Norbert Stadler, Datenschutz durch Organisation

- Voraussetzung des Datenschutzes (Gerhard Stadler) . . . . . . . . . . . 371

Colin Tapper, Computers and the Law (Petra Wuttke-Götz) . . . . . . . . . . 365

Klaus Tiedemann/Christoph Sasse, Delinquenzprophylaxe, Kreditsicherung und Datenschutz in der Wirtschaft (Ruprecht Kamlah) . . . . . . . . . . . . 195

Jürgen Welp, Die strafprozessuale Überwachung des Post- und

Fernmeldeverkehrs (Sigmar Uhlig)

Klaus-G. Wendt, Informationsbedarf für industrielles Management (Sigmar Uhlig) 372

\title{
Die Autoren der Beiträge
}

Ulrich Bechmann, GMD Institut für Datenverarbeitung im Rechtswesen, SchloB Birlinghoven, Postfach 1240, 5205 St. Augustin 1

Hélène Bauer-Bernet, Dr. jur., Lic en Droit, Lic es Lettres, 11 Drève des Deux Moutiers, Brüssel

Herbert Burkert, Konviktsweg 18, D-6110 Dieburg

Ulrich Dammann, Wissenschaftlicher Mitarbeiter der Forschungsstelle für Juristische Dokumentation, Senckenberganlage 31, D-6000 Frankfurt

Christine Falke, Gustav-Adolf-Str. 9, D-4800 Bielefeld 1

Hansjürgen Garstka, Dr., Assistenzprofessor, Freie Universität Berlin, Fachbereich Rechtswissenschaft, Corneliusstr. 20, D-1000 Berlin 46

Andrzej Kisza, Dr. jur. habil., Rechtsanwalt, Wroclaw 50-950 Polen, ul. Sadowa 4 (Zespol Adwokacki $\mathrm{nr}$ 4)

Henriette Mignot, CRIDON, 58, bvd. des Belges, F-69 458 Lyon

Paul J. Müller, Diplom-Volkswirt, Zentralarchiv für empirische Sozialforschung der Universität Köln, Bachemer Str. 40, D-5000 Köln

Walter Popp. Technische Hochschule Darmstadt, Fachgebiet für Offentliches Recht, Hochschulstr. 1/III, D-6100 Darmstadt

Leo Reisinger, DDr. Privatdozent, Institut für Statistik an der Universität Wien, Universitätsstr. 7, A-1010 Wien

Hubert Rodingen, Reinhold-Schneider-Str. 1, D-6500 Mainz-Gonsenheim

Maria Schlagböhmer, Richterin am Oberlandesgericht, Bundesministerium der Justiz, Stresemannstr. 6, D-5300 Bonn - Bad Godesberg

Bernhard Schlink, Technische Hochschule Darmstadt, Fachgebiet für Offentliches Recht, Hochschulstr. 1/III, D-6100 Darmstadt

Ulrich Seidel, Dr., GMD Institut für Datenverarbeitung im Rechtswesen, SchloB Birlinghoven, Postfach 1240, D-5205 St. Augustin 1

Dieter Zimmermann, Unternehmens-Berater, Kaiserwerther Str. 129, D-4000 Düsseldorf 


\section{Hansjürgen Garstka}

\section{Information und Sprache im rechtlichen Regelprozeß. Zur Einbeziehung von Informationswissenschaft und Sprachwissenschaft in der Rechtstheorie}

\author{
Übersicht \\ 1 Ausgangspunkt \\ 2 Die juristische Entscheidung \\ 3 Informationswissenschaft und Recht
}

\author{
4 Sprachwissenschaft und Recht \\ Zusammenfassung
}

\section{Ausgangspunkt}

Die derzeitige Entwicklung der Rechtstheorie in der BRD ist gekennzeichnet durch eine zunehmende Hinwendung zu Forschungsgebieten, die außerhalb der Rechtswissenschaft liegen, denen man aber bei der Suche nach einer rationalen Theorie des Rechts große Bedeutung zumißt. Insbesondere soll die Einführung „exakter" Wissenschaftsdisziplinen die Rationalität der Rechtstheorie und damit auch der Rechtswissenschaft insgesamt erhöhen'.

Diese Zielsetzung hat auch einen praktischen Grund: In immer stärkerem $\mathrm{Maß}$ werden Überlegungen angestellt, wie Elektronische Datenverarbeitungsanlagen in die primär rechtlich strukturierten gesellschaftlichen Bereiche eingeführt werden können. Hier setzt die Automation Reflexionen über die algorithmische Struktur und somit eine exakte Analyse juristischer Handlungsabläufe voraus.

Informationswissenschaft und Sprachwissenschaft sind zwei Disziplinen, die einen bedeutenden Beitrag zur Weiterung der Rechtstheorie in dieser Hinsicht leisten können.

Ihre Einführung in die Rechtstheorie ist allerdings mit einer Neubesinnung über den Gegenstandsbereich der Rechtstheorie selbst verbunden. Die traditionelle Rechtstheorie in der BRD (und nicht nur hier) hat sich bis vor kurzem nahezu ausschließlich mit der isolierten Betrachtung der in der jeweiligen Gesellschaft geltenden Rechtsordnung zufrieden gegeben. Dabei wird die Rechtsordnung verstanden als die Menge der Rechtsnormen, die in den Gesetzen niedergelegten Rechtssätzen repräsentiert sind und zu denen Rechtsprechung und wissenschaftliche Literatur als zusätzliche Erkenntnisquellen hinzutreten. Im Vordergrund steht die Frage, wie bei unklarem Wortlaut oder bei Lücken im Recht eine Entscheidung für eine der möglichen Deutungen gefunden werden kann: so kommt es mitunter zur Identifikation von Juristischer Methodenlehre und Rechts-

\footnotetext{
' Vgl hierzu vor allem den Sammelband: Rechtstheorie als Grundlagenwissenschaft der Rechtswissenschaft (herausgegeben von H. Albert, N. Luhmann, W. Maihofer, O. Weinberger). Düsseldorf 1972. Jahrbuch für Rechtssoziologie und Rechtstheorie. Band 2.
} 